TRANSACTIONS OF THE

AMERICAN MATHEMATICAL SOCIETY

Volume 364, Number 6, June 2012, Pages 3329-3347

S 0002-9947(2012)05607-8

Article electronically published on January 31,2012

\title{
STRICHARTZ ESTIMATES FOR DIRICHLET-WAVE EQUATIONS IN TWO DIMENSIONS WITH APPLICATIONS
}

\author{
HART F. SMITH, CHRISTOPHER D. SOGGE, AND CHENGBO WANG
}

\begin{abstract}
We establish the Strauss conjecture for nontrapping obstacles when the spatial dimension $n$ is two. As pointed out by Hidano, Metcalfe, Smith, Sogge, and Zhou (2010) this case is more subtle than $n=3$ or 4 due to the fact that the arguments in the papers of the first two authors (2000), Burq (2000) and Metcalfe (2004), showing that local Strichartz estimates for obstacles imply global ones, require that the Sobolev index, $\gamma$, equals $1 / 2$ when $n=2$. We overcome this difficulty by interpolating between energy estimates $(\gamma=0)$ and ones for $\gamma=\frac{1}{2}$ that are generalizations of Minkowski space estimates of Fang and the third author (2006), (2011), the second author (2008) and Sterbenz (2005).
\end{abstract}

\section{INTRODUCTION}

In a recent series of papers, [3, 7, techniques have been developed to prove general Strichartz estimates for wave equations outside of nontrapping obstacles. These papers relied on ideas that were used to prove the more standard $L_{t}^{q} L_{x}^{r}$ Strichartz estimates for obstacles in [1, [9] and [11. As was shown in [7, though, a limitation arises in the proof which is only relevant when the spatial dimension, $n$, equals two. This is, that the $T T^{*}$ arguments involving the Christ-Kiselev lemma [2] a priori require that the Sobolev regularity for the data in the homogeneous estimates be equal to $\frac{1}{2}$ when $n=2$, with similar restrictions on the estimates for the inhomogeneous wave equation.

As we shall see in this paper, even though we can only directly prove Strichartz estimates involving Sobolev regularity of $\gamma=\frac{1}{2}$, for some applications if we interpolate with trivial (energy) estimates, this is enough. In particular, we shall be able to establish the Strauss conjecture for obstacles when $n=2$. Specifically, if $\mathcal{K} \subset \mathbb{R}^{2}$ is a compact nontrapping obstacle with smooth boundary, then we shall be able to show that there are global small-amplitude solutions of the equation

$$
\left\{\begin{array}{l}
\square u(t, x)=F_{p}(u(t, x)), \quad(t, x) \in \mathbb{R}_{+} \times \mathbb{R}^{2} \backslash \mathcal{K}, \\
u(t, x)=0, \quad x \in \partial \mathcal{K}, \\
\left.u\right|_{t=0}=f,\left.\quad \partial_{t} u\right|_{t=0}=g
\end{array}\right.
$$

provided that

$$
\left|F_{p}(u)\right|+|u|\left|F_{p}^{\prime}(u)\right| \lesssim|u|^{p}, \quad \text { for }|u| \leq 1,
$$

Received by the editors December 14, 2010 and, in revised form, April 17, 2011.

2010 Mathematics Subject Classification. Primary 35L71; Secondary 35B45, 35L20.

Key words and phrases. Strichartz estimates, Strauss conjecture, obstacles.

The authors were supported in part by the NSF. The third author was supported in part by NSFC 10871175 and 10911120383.

(C)2012 American Mathematical Society Reverts to public domain 28 years from publication 
and $p$ is larger than the critical exponent for (1.1) when $n=2$, which is $p_{c}=$ $(3+\sqrt{17}) / 2$, or equivalently

$$
p^{2}-3 p-2>0 \text { and } p>0 \text {. }
$$

Fortunately, the critical family of estimates that we require for proving bounds for this equation involve $\gamma=\frac{1}{2}$ (see Figure 11). All the other estimates, including the ones we shall use, come from interpolating between these and energy estimates.

Let us state our existence results for (1.1) with more precision. We first introduce some notation. We will denote

$$
\Omega=\mathbb{R}^{2} \backslash \mathcal{K}
$$

and let $\dot{H}^{\gamma}(\Omega)$ be the homogeneous Sobolev space of order $\gamma$ on $\Omega$, with norm

$$
\|f\|_{\dot{H}^{\gamma}(\Omega)}=\left\|\left(\sqrt{-\Delta_{D}}\right)^{\gamma} f\right\|_{L^{2}(\Omega)}
$$

with $\Delta_{D}$ the Dirichlet-Laplacian in $\Omega$. If $0 \leq \gamma<\frac{1}{2}$, then for $f \in C^{\infty}(\Omega)$ we have

$$
\|f\|_{\dot{H}^{\gamma}(\Omega)} \approx\|\tilde{f}\|_{\dot{H}^{\gamma}\left(\mathbb{R}^{2}\right)},
$$

if $\tilde{f}(x)=f(x), x \in \Omega$ and $\tilde{f}(x)=0, x \in \mathcal{K}$. Here $\dot{H}^{\gamma}\left(\mathbb{R}^{2}\right)$ denotes the homogeneous Sobolev space with norm

$$
\|g\|_{\dot{H}^{\gamma}\left(\mathbb{R}^{2}\right)}^{2}=\left.\left.(2 \pi)^{-2} \int_{\mathbb{R}^{2}}|| \xi\right|^{\gamma} \hat{g}(\xi)\right|^{2} d \xi
$$

with $\hat{g}$ denoting the Fourier transform of $g$. See also the introduction of [7] for a discussion of the space $\dot{H}^{\gamma}(\Omega)$.

If we also let $\partial_{j}=\partial_{x_{j}}, j=1,2$, and

$$
\{Z\}=\left\{\partial_{1}, \partial_{2}, x_{1} \partial_{2}-x_{2} \partial_{1}\right\}
$$

then we can state our existence theorem for (1.1). The norm used in (1.5) is certainly not the best possible; see the remarks following Corollary 3.3

Theorem 1.1. Let $n=2$ and $\mathcal{K}, \Omega$ be as above. If $p_{c}<p<5$, then there is an $\varepsilon_{0}=\varepsilon_{0}(p, \Omega)>0$ such that (1.1) has a global solution satisfying

$$
Z^{\alpha} u(t, \cdot) \in L^{p-1}(\Omega), \quad|\alpha| \leq 1,
$$

provided that the initial data $(f, g)=\left(\left.u\right|_{t=0},\left.\partial_{t} u\right|_{t=0}\right)$ satisfies $\left.f\right|_{\partial \Omega}=0$ and

$$
\sum_{|\alpha| \leq 2}\left\|Z^{\alpha} f\right\|_{L^{q_{p}}(\Omega)}+\sum_{|\alpha| \leq 1}\left\|Z^{\alpha} g\right\|_{L^{q_{p}}(\Omega)}<\varepsilon, \quad 0<\varepsilon<\varepsilon_{0},
$$

with $\frac{1}{q_{p}}=\frac{1}{p-1}+\frac{1}{2}$. If $p \geq 5$, then there is a global solution of (1.1) if (1.5) holds with $q=q_{\tilde{p}}$, for some $\tilde{p} \in\left(p_{c}, 5\right)$.

Note that by Sobolev embedding

$$
\begin{aligned}
& \sum_{|\alpha| \leq 1}\left(\left\|Z^{\alpha} f\right\|_{\dot{H}^{\gamma_{p}}(\Omega)}+\left\|Z^{\alpha} g\right\|_{\dot{H}^{\gamma_{p}-1}(\Omega)}\right) \\
& \quad \leq C_{p}\left(\sum_{|\alpha| \leq 2}\left\|Z^{\alpha} f\right\|_{L^{q_{p}}(\Omega)}+\sum_{|\alpha| \leq 1}\left\|Z^{\alpha} g\right\|_{L^{q_{p}}(\Omega)}\right),
\end{aligned}
$$

where $q_{p}$ is as above and

$$
\gamma_{p}=1-\frac{2}{p-1}
$$


is the scaling exponent for the equation $\square u=|u|^{p}$ in two dimensions (see, e.g. [12]). In earlier works $([3,7])$ the smallness assumption on the data was based on the size of the $\dot{H}^{\gamma_{p}}(\Omega) \times \dot{H}^{\gamma_{p}-1}(\Omega)$ norm of derivatives of $(f, g)$ (see also (2.19) below). For technical reasons, we are led to making the somewhat stronger assumption (1.5) involving the $L^{q_{p}}$ norms, but this too is natural.

To prove Theorem 1.1 we shall require certain Strichartz estimates in $\Omega$. We shall postpone formulating them until they are needed in $\S 3$, but they are related to the following 2-dimensional Minkowski space estimates, which involve the angular mixed-norm spaces

$$
\|f\|_{L_{|x|}^{r} L_{\theta}^{2}\left(\mathbb{R}^{2}\right)}=\left(\int_{0}^{\infty}\left(\int_{0}^{2 \pi}|f(\rho(\cos \theta, \sin \theta))|^{2} d \theta\right)^{r / 2} \rho d \rho\right)^{1 / r} .
$$

Proposition 1.2. Let $P=\sqrt{-\Delta}$ in $\mathbb{R}^{2}$. Assume that $(q, r) \neq(\infty, \infty)$,

$$
q, r>2 \text { and } \frac{1}{q}<\frac{1}{2}-\frac{1}{r}
$$

or $(q, r)=(\infty, 2)$. Then

$$
\left\|e^{-i t P} g\right\|_{L_{t}^{q} L_{|x|}^{r} L_{\theta}^{2}\left(\mathbb{R} \times \mathbb{R}^{2}\right)} \leq C_{q, r}\|g\|_{\dot{H}^{\gamma}\left(\mathbb{R}^{2}\right)}, \quad \gamma=2\left(\frac{1}{2}-\frac{1}{r}\right)-\frac{1}{q} .
$$

See Figure 1 for the range of exponents in (1.8):

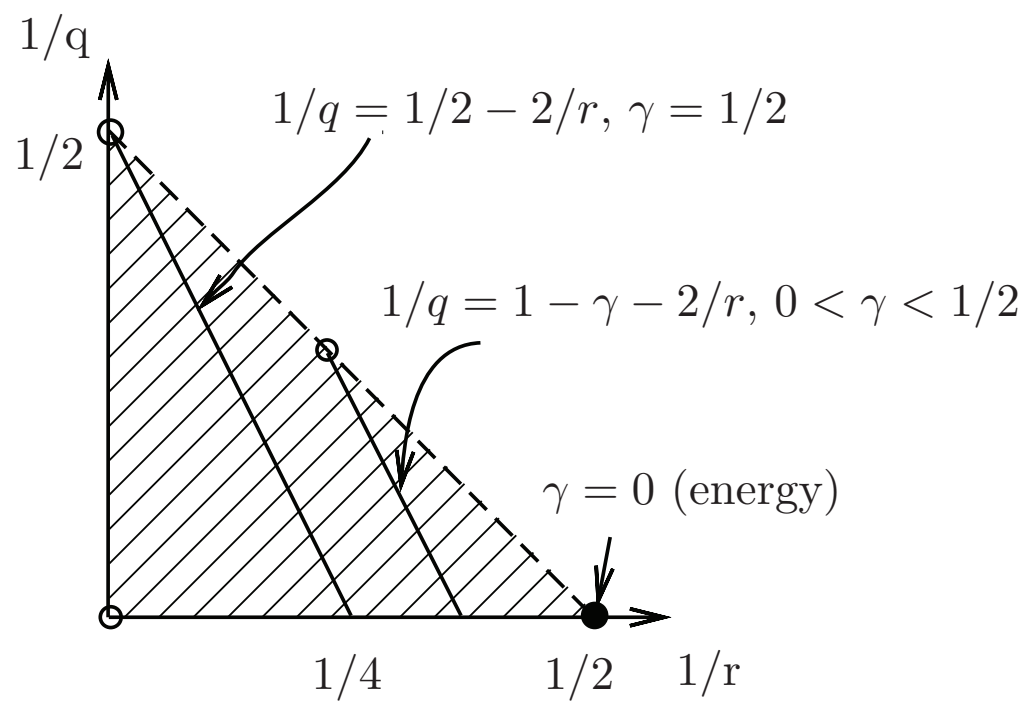

FigurE 1. Minkowski space exponents

We mention that Sterbenz [14] proved related estimates where $L_{\theta}^{2}$ is replaced by $L_{\theta}^{r}$ (with norms of different regularity on the right). Related results are also due to Fang and Wang [4, [5] and to Sogge [12] (for $n=3$ ). Since the proof of (1.8) is simple, we shall present it in $\S 2$ for the sake of completeness. It can be adapted to give a slightly different proof of the corresponding results in [14] and [5]. 
This paper is organized as follows. In the next section, we shall prove Proposition 1.2. We shall also show how it can be used to give a simple proof of Glassey's theorem [6] which says that the Strauss conjecture holds for $\mathbb{R}_{+} \times \mathbb{R}^{2}$, since this will serve as a model for the more technical arguments that are needed to establish Theorem 1.1. In the final section, we shall formulate and prove the variants of (1.8) that we require and then present the proof of this theorem.

\section{Estimates for $\mathbb{R}_{+} \times \mathbb{R}^{2}$ And Glassey's theOrem}

We shall first prove Proposition 1.2 and then give the simple argument showing how it can be used to prove Glassey's theorem that in $\mathbb{R}_{+} \times \mathbb{R}^{2}$ there is small amplitude global existence for $\square u=|u|^{p}$ when $p>p_{c}=(3+\sqrt{17}) / 2$.

The main step in the proof of (1.8) will be to show that

$$
\left\|e^{-i t P} f\right\|_{L_{t}^{q} L_{|x|}^{\infty} L_{\theta}^{2}\left(\mathbb{R} \times \mathbb{R}^{2}\right)} \leq C_{q}\|f\|_{L^{2}\left(\mathbb{R}^{2}\right)} \text { if } q>2 \text {, and } \hat{f}(\xi)=0 \text { if }|\xi| \notin\left[\frac{1}{2}, 1\right] .
$$

Since Hardy-Littlewood-Sobolev estimates give $\dot{H}^{1-\frac{2}{r}}\left(\mathbb{R}^{2}\right) \subset L^{r}\left(\mathbb{R}^{2}\right), 2 \leq r<\infty$, we also clearly have

$$
\left\|e^{-i t P} f\right\|_{L_{t}^{\infty} L_{|x|}^{r} L_{\theta}^{2}} \leq C_{r}\|f\|_{\dot{H}^{1-\frac{2}{r}}}
$$

since $e^{-i t P}$ is a unitary operator on $\dot{H}^{\gamma}$. The estimates (2.1) and (2.2) say that we have the estimates described in Figure 1 that respectively correspond to the (open) vertical and (half open) horizontal segments. By interpolation we conclude that, if $q, r>2$ and $\frac{1}{q}<\frac{1}{2}-\frac{1}{r}$, then

$$
\left\|e^{-i t P} f\right\|_{L_{t}^{q} L_{|x|}^{r} L_{\theta}^{2}} \leq C_{q, r}\|f\|_{L^{2}\left(\mathbb{R}^{2}\right)} \quad \text { if } \hat{f}(\xi)=0,|\xi| \notin\left[\frac{1}{2}, 1\right] .
$$

By scaling and Littlewood-Paley theory, we obtain from this that if we remove the support assumptions on the Fourier transform, then for $q$ and $r$ as above, and $(q, r) \neq(\infty, \infty)$

$$
\left\|e^{-i t P} g\right\|_{L_{t}^{q} L_{|x|}^{r} L_{\theta}^{2}\left(\mathbb{R} \times \mathbb{R}^{2}\right)} \leq C_{q, r}\|g\|_{\dot{H}^{1-\frac{2}{r}-\frac{1}{q}}\left(\mathbb{R}^{2}\right)},
$$

which is the inequality in Proposition 1.2

Let us turn to the proof of (2.1). By the support assumptions for $\hat{f}$ we have that

$$
\|f\|_{L^{2}\left(\mathbb{R}^{2}\right)}^{2} \approx \int_{0}^{\infty} \int_{0}^{2 \pi}|\hat{f}(\rho(\cos \omega, \sin \omega))|^{2} d \omega d \rho .
$$

We expand the angular part of $\hat{f}$ using Fourier series and find that if $\xi=$ $\rho(\cos \omega, \sin \omega)$, then there are coefficients $c_{k}(\rho)$ which vanish for $\rho \notin\left[\frac{1}{2}, 1\right]$ so that

$$
\hat{f}(\xi)=\sum_{k} c_{k}(\rho) e^{i k \omega}
$$

By (2.4) and Plancherel's theorem for $S^{1}$ and $\mathbb{R}$, we have

$$
\|f\|_{L^{2}\left(\mathbb{R}^{2}\right)}^{2} \approx \sum_{k} \int_{\mathbb{R}}\left|c_{k}(\rho)\right|^{2} d \rho \approx \sum_{k} \int_{\mathbb{R}}\left|\hat{c}_{k}(s)\right|^{2} d s,
$$

where $\hat{c}_{k}(s), s \in \mathbb{R}$, denotes the one-dimensional Fourier transform of $c_{k}(\rho)$. Recall that (see Stein and Weiss [13, p. 137)

$$
f(r(\cos \omega, \sin \omega))=(2 \pi)^{-1} \sum_{k}\left(i^{k} \int_{0}^{\infty} J_{k}(r \rho) c_{k}(\rho) \rho d \rho\right) e^{i k \omega}
$$


where $J_{k}, k \in \mathbb{Z}$, is the $k$-th Bessel function defined by

$$
J_{k}(y)=\frac{(-i)^{k}}{2 \pi} \int_{0}^{2 \pi} e^{i y \cos \theta-i k \theta} d \theta .
$$

By (2.6) and the support properties of the $c_{k}$, if we fix $\beta \in C_{0}^{\infty}(\mathbb{R})$ satisfying $\beta(\tau)=1$ for $\tau \in\left[\frac{1}{2}, 1\right]$ and $\beta(\tau)=0$ for $\tau \notin\left[\frac{1}{4}, 2\right]$, then with $\alpha(\rho)=\rho \beta(\rho) \in \mathcal{S}(\mathbb{R})$, we have

$$
\begin{aligned}
\left(e^{-i t P} f\right) & (r(\cos \omega, \sin \omega)) \\
& =(2 \pi)^{-1} \sum_{k}\left(i^{k} \int_{0}^{\infty} J_{k}(r \rho) e^{-i t \rho} c_{k}(\rho) \beta(\rho) \rho d \rho\right) e^{i k \omega} \\
& =(2 \pi)^{-2} \sum_{k}\left(i^{k} \int_{0}^{\infty} \int_{-\infty}^{\infty} J_{k}(r \rho) e^{i \rho(s-t)} \hat{c}_{k}(s) \alpha(\rho) d s d \rho\right) e^{i k \omega} \\
& =(2 \pi)^{-3} \sum_{k}\left(\int_{0}^{\infty} \int_{-\infty}^{\infty} \int_{0}^{2 \pi} e^{i \rho r \cos \theta} e^{-i k \theta} e^{i \rho(s-t)} \hat{c}_{k}(s) \alpha(\rho) d \theta d s d \rho\right) e^{i k \omega} \\
& =(2 \pi)^{-3} \sum_{k}\left(\int_{-\infty}^{\infty} \int_{0}^{2 \pi} e^{-i k \theta} \hat{\alpha}((t-s)-r \cos \theta) \hat{c}_{k}(s) d \theta d s\right) e^{i k \omega} .
\end{aligned}
$$

As a result, we have that for any $r \geq 0$,

$$
\begin{aligned}
& \int_{0}^{2 \pi}\left|\left(e^{-i t P} f\right)(r(\cos \omega, \sin \omega))\right|^{2} d \omega \\
& =(2 \pi)^{-5} \sum_{k}\left|\int_{-\infty}^{\infty} \int_{0}^{2 \pi} e^{-i k \theta} \hat{\alpha}((t-s)-r \cos \theta) \hat{c}_{k}(s) d \theta d s\right|^{2} .
\end{aligned}
$$

To estimate the right side we shall use the following.

Lemma 2.1. Let $\alpha \in \mathcal{S}(\mathbb{R})$ and $N \in \mathbb{N}$ be fixed. Then there is a uniform constant $C$, which is independent of $m \in \mathbb{R}$ and $r \geq 0$, so that the following inequalities hold. First,

$$
\int_{0}^{2 \pi}|\alpha(m-r \cos \theta)| d \theta \leq C\langle m\rangle^{-N} \quad \text { if } 0 \leq r \leq 1 \text { or }|m| \geq 2 r .
$$

If $r>1$ and $|m| \leq 2 r$, then

$$
\int_{0}^{2 \pi}|\alpha(m-r \cos \theta)| d \theta \leq C\left(r^{-1}+r^{-\frac{1}{2}}\langle r-|m|\rangle^{-\frac{1}{2}}\right) .
$$

Consequently, if $\delta>0$, there is a constant $A_{\delta}$, which is independent of $t \in \mathbb{R}$ and $r \geq 0$, so that

$$
\int_{-\infty}^{\infty}\left(\int_{0}^{2 \pi}\langle t-s\rangle^{\frac{1}{2}-\delta}|\alpha((t-s)-r \cos \theta)| d \theta\right)^{2} d s \leq A_{\delta} .
$$

If we apply (2.11) and (2.8) along with the Schwarz inequality, we conclude that if $f$ is as in (2.1), then for $\delta>0$

$$
\left\|e^{-i t P} f\right\|_{L_{|x|}^{\infty} L_{\theta}^{2}}^{2} \leq B_{\delta} \sum_{k} \int_{-\infty}^{\infty}\left|\langle t-s\rangle^{-\frac{1}{2}+\delta} \hat{c}_{k}(s)\right|^{2} d s,
$$

which, by Minkowski's inequality and (2.5), in turn yields (2.1). 
Proof of Lemma 2.1. We first realize that inequalities (2.9) and (2.10) clearly imply (2.11). Also, (2.9) is trivial since $\alpha \in \mathcal{S}$. Therefore, we just need to prove (2.10). To do so, it suffices to show that

$$
\int_{0}^{\pi / 4}|\alpha(m-r \cos \theta)| d \theta+\int_{\pi-\pi / 4}^{\pi}|\alpha(m-r \cos \theta)| d \theta \leq C r^{-\frac{1}{2}}\langle r-|m|\rangle^{-\frac{1}{2}}
$$

and also

$$
\int_{\pi / 4}^{\pi-\pi / 4}|\alpha(m-r \cos \theta)| d \theta \leq C r^{-1}
$$

In order to prove (2.12), it suffices to prove that the first integral is controlled by the right side. If we apply this estimate to the function $\alpha(-s)$, we then see that the second integral satisfies the same bounds. We can estimate the first integral if we make the substitution $u=1-\cos \theta$, in which case we see that it equals

$$
\begin{aligned}
\int_{0}^{1-1 / \sqrt{2}}|\alpha((m-r)+r u)| \frac{d u}{\sqrt{2 u-u^{2}}} & \leq \int_{0}^{1-1 / \sqrt{2}}|\alpha((m-r)+r u)| \frac{d u}{\sqrt{u}} \\
& \leq C r^{-\frac{1}{2}} \int_{0}^{\infty}|\alpha((m-r)+u)| \frac{d u}{\sqrt{u}} \\
& \leq C^{\prime} r^{-\frac{1}{2}}\langle r-|m|\rangle^{-\frac{1}{2}},
\end{aligned}
$$

as desired, which completes the proof of (2.12).

To prove (2.13) we just make the change of variables $u=r \cos \theta$ and note that $|d u / d \theta| \approx r$ on the region of integration, which leads to the inequality as $\alpha \in \mathcal{S}$.

We conclude this section by showing how Proposition 1.2 implies estimates that can be used to prove Glassey's [6] existence theorem for $\square u=|u|^{p}$ when $n=2$. Specifically, if $u$ solves the wave equation for $\mathbb{R} \times \mathbb{R}^{2}$,

$$
\left\{\begin{array}{l}
\square u=F, \\
\left.u\right|_{t=0}=f,\left.\quad \partial_{t} u\right|_{t=0}=g,
\end{array}\right.
$$

then

$$
\|u\|_{L_{t}^{q} L_{|x|}^{r} L_{\theta}^{2}}+\|u\|_{L_{t}^{\infty} \dot{H}^{\gamma}} \lesssim\|f\|_{\dot{H}^{\gamma}}+\|g\|_{\dot{H}^{\gamma-1}}+\|F\|_{L_{t}^{\tilde{q}^{\prime}} L_{|x|}^{\tilde{r}^{\prime} \mid} L_{\theta}^{2}},
$$

assuming that $q, r, \tilde{q}, \tilde{r}>2$ with $(q, r),(\tilde{q}, \tilde{r}) \neq(\infty, \infty), \frac{1}{q}<\frac{1}{2}-\frac{1}{r}, \frac{1}{\tilde{q}}<\frac{1}{2}-\frac{1}{\tilde{r}}$, and

$$
\gamma=1-\frac{2}{r}-\frac{1}{q}, \quad \text { and } 1-\gamma=1-\frac{2}{\tilde{r}}-\frac{1}{\tilde{q}} .
$$

In (2.15), $\tilde{q}^{\prime}$ and $\tilde{r}^{\prime}$ denote the exponents which are conjugate to $\tilde{q}$ and $\tilde{r}$, respectively, and also, here and in what follows, the space-time norms are taken over $\mathbb{R}_{+} \times \mathbb{R}^{2}$. Clearly, (2.15) follows from (1.8) and energy estimates if the forcing term, $F$, in (2.14) vanishes. Since we are assuming (2.16) and since $\tilde{q}^{\prime}<q$, the estimates for the inhomogeneous wave equation follow from an application of the Christ-Kiselev lemma 2] (cf. [12, pp. 136-141). 
If $\{Z\}$ are the operators in (1.4), then since they commute with $\square$, (2.15) implies that

$$
\begin{aligned}
\sum_{|\alpha| \leq 1}\left(\left\|Z^{\alpha} u\right\|_{L_{t}^{q} L_{|x|}^{r} L_{\theta}^{2}}+\left\|Z^{\alpha} u\right\|_{L_{t}^{\infty} \dot{H}^{\gamma}}\right) & \\
& \lesssim \sum_{|\alpha| \leq 1}\left(\left\|Z^{\alpha} f\right\|_{\dot{H}^{\gamma}}+\left\|Z^{\alpha} g\right\|_{\dot{H}^{\gamma-1}}+\left\|Z^{\alpha} F\right\|_{L_{t}^{\tilde{q}^{\prime} L_{|x|}^{\tilde{r}^{\prime}} L_{\theta}^{2}}}\right),
\end{aligned}
$$

with $q, r, \tilde{q}^{\prime}, \tilde{r}^{\prime}$ and $\gamma$ as above. Let us now present the simple argument showing that this estimate implies that there are global solutions of the equation

$$
\left\{\begin{array}{l}
\square u(t, x)=F_{p}(u(t, x)), \quad(t, x) \in \mathbb{R}_{+} \times \mathbb{R}^{2}, \\
\left.u\right|_{t=0}=f,\left.\quad \partial_{t} u\right|_{t=0}=g,
\end{array}\right.
$$

if $F_{p}$ is as in (1.2), with $p$ as in (1.3), assuming that (when $p_{c}<p<5$ ) the initial data satisfies

$$
\sum_{|\alpha| \leq 1}\left(\left\|Z^{\alpha} f\right\|_{\dot{H}^{\gamma_{p}}}+\left\|Z^{\alpha} g\right\|_{\dot{H}^{\gamma_{p}-1}}\right)<\varepsilon, \quad \gamma_{p}=1-\frac{2}{p-1},
$$

with $\varepsilon=\varepsilon(p)$ sufficiently small.

We first consider the subconformal range where $\frac{3+\sqrt{17}}{2}=p_{c}<p<5$. This range easily lends itself to the special case of (2.17), which says that, for such $p$,

$$
\begin{aligned}
& \sum_{|\alpha| \leq 1}\left(\left\|Z^{\alpha} u\right\|_{L_{t} \frac{(p-1) p}{2}}^{\frac{\left(L_{|x|}^{p} L_{\theta}^{2}\right.}{2}}+\left\|Z^{\alpha} u\right\|_{L_{t}^{\infty} \dot{H}^{\gamma_{p}}}\right) \\
& \lesssim \sum_{|\alpha| \leq 1}\left(\left\|Z^{\alpha} f\right\|_{\dot{H}^{\gamma_{p}}}+\left\|Z^{\alpha} g\right\|_{\dot{H}^{\gamma_{p}-1}}+\left\|Z^{\alpha} F\right\|_{L_{t}^{\frac{p-1}{2}} L_{|x|}^{1} L_{\theta}^{2}}\right) .
\end{aligned}
$$

The temporary assumption that $p<5$ is needed to ensure that $(p-1) / 2<2$, and, therefore, $[(p-1) / 2]^{\prime}>2$, which is the first part of the assumptions for (2.15). The more serious assumption that $p>p_{c}$, which is (1.3), is equivalent to the second part of (1.7) for the exponents on the left side of (2.20). That is, for $p>0$,

$$
\frac{2}{p(p-1)}<\frac{1}{2}-\frac{1}{p} \Longleftrightarrow p>p_{c} .
$$

Using (2.20), we shall show that we can solve (2.18) by an iteration argument for $p_{c}<p<5$, provided that $\varepsilon>0$ in (2.19) is small. To be more specific, we shall let $u_{0}$ solve the Cauchy problem (2.14) with $F \equiv 0$. We then iteratively define $u_{k}$, $k \geq 1$, by solving

$$
\left\{\begin{array}{l}
\square u_{k}(t, x)=F_{p}\left(u_{k-1}(t, x)\right), \quad(t, x) \in \mathbb{R}_{+} \times \mathbb{R}^{2}, \\
\left.u_{k}\right|_{t=0}=f,\left.\quad \partial_{t} u_{k}\right|_{t=0}=g .
\end{array}\right.
$$

Our aim is to show that if $\varepsilon>0$ in (2.19) is small enough, then

$$
M_{k}=\sum_{|\alpha| \leq 1}\left(\left\|Z^{\alpha} u_{k}\right\|_{L_{t}^{\frac{(p-1) p}{2}} L_{|x|}^{p} L_{\theta}^{2}}+\left\|Z^{\alpha} u_{k}\right\|_{L_{t}^{\infty} \dot{H}^{\gamma_{p}}}\right)
$$

must also be small. 
For $k=0$, it follows from (2.20) that $M_{0} \leq C_{0} \varepsilon$, with $C_{0}$ a fixed constant. Clearly, (2.20) also yields that for $k=1,2,3, \ldots$,

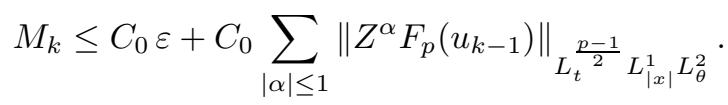

To control the last term, we note that our assumption (1.2) on $F_{p}$ implies that

$$
\sum_{|\alpha| \leq 1}\left|Z^{\alpha} F_{p}(v)\right| \lesssim|v|^{p-1} \sum_{|\alpha| \leq 1}\left|Z^{\alpha} v\right| \quad \text { if }|v| \leq 1
$$

Since $\partial_{\theta}=x_{1} \partial_{2}-x_{2} \partial_{1} \in\{Z\}$, we have

$$
\|v(|x| \cdot)\|_{L_{\theta}^{\infty}} \lesssim \sum_{|\alpha| \leq 1}\left\|Z^{\alpha} v(|x| \cdot)\right\|_{L_{\theta}^{2}}
$$

and since $0<\gamma_{p}<1$ and $\partial_{j} \in\{Z\}, j=1,2$, Sobolev estimates imply that

$$
\|v\|_{L^{\infty}\left(\mathbb{R}^{2}\right)} \lesssim \sum_{|\alpha| \leq 1}\left\|Z^{\alpha} v\right\|_{\dot{H}^{\gamma_{p}}\left(\mathbb{R}^{2}\right)}
$$

so that (1.2) applies in our case. Combining the above inequalities gives

$$
M_{k} \leq C_{0} \varepsilon+C_{1} C_{0} M_{k-1}^{p},
$$

for some uniform constant $C_{1}$. Since $M_{0} \leq C_{0} \varepsilon$, we deduce from this that if $\varepsilon>0$ is sufficiently small, then

$$
M_{k} \leq 2 C_{0} \varepsilon, \quad k=1,2,3, \ldots
$$

To finish the proof of the existence results for $p_{c}<p<5$, it suffices to show that

$$
A_{k}=\left\|u_{k}-u_{k-1}\right\|_{L_{t} \frac{(p-1) p}{2}} L_{|x|}^{p} L_{\theta}^{2}
$$

tends geometrically to zero as $k \rightarrow \infty$. Since $\left|F_{p}(w)-F_{p}(v)\right| \lesssim|v-w| \cdot\left(|v|^{p-1}+\right.$ $|w|^{p-1}$ ) when $|v|,|w| \leq 1$, the proof of (2.22) can be adapted to show that, for small $\varepsilon>0$, there is a uniform constant $C$ so that

$$
A_{k} \leq C A_{k-1}\left(M_{k-1}+M_{k-2}\right)^{p-1}
$$

which, by (2.22), implies that $A_{k} \leq \frac{1}{2} A_{k-1}$ for small $\varepsilon>0$. Since $A_{1}$ is finite, the claim follows, which finishes the proof of the existence results for $p_{c}<p<5$.

As we noted above, we cannot directly get the existence results from (2.20) if $p \geq 5$. However, since the assumptions (1.2) on $F_{p}$ become weaker with increasing $p$, the above argument yields existence results for this case as well.

\section{The Strauss COnjecture for nOntrapping ObStacles} IN 2-DIMENSIONS

The goal of this section is to show that we can solve the semilinear Dirichletwave equation (1.1) for small data when $\mathcal{K} \subset \mathbb{R}^{2}$ is a nontrapping obstacle and, as in (1.3), $p>p_{c}=\frac{3+\sqrt{17}}{2}$. The main step will be to find a suitable variant of the Minkowski space estimate (1.8) which is valid for solutions of the linear Dirichlet-wave equation

$$
\left\{\begin{array}{l}
\square u(t, x)=F(t, x), \quad(t, x) \in \mathbb{R}_{+} \times \Omega, \\
u(t, x)=0, \quad(t, x) \in \mathbb{R}_{+} \times \partial \Omega, \\
\left.u\right|_{t=0}=f,\left.\partial_{t} u\right|_{t=0}=g
\end{array}\right.
$$


where, as before, $\Omega=\mathbb{R}^{2} \backslash \mathcal{K}$. As previously noted, we are in luck because the crucial estimates for (1.8) involve Sobolev regularity of $\gamma=\frac{1}{2}$, which is the regularity necessary for $n=2$ to use the techniques of [7, [1, 9] and [1], to show that local in time Strichartz estimates for $\Omega$, coupled with global in time estimates for $\mathbb{R}^{2}$, imply global in time estimates for $\Omega$. After we obtain these estimates for $\gamma=\frac{1}{2}$, we shall be able to obtain a family of estimates corresponding to other $\gamma$ by interpolating with energy estimates. The range of exponents will be slightly smaller than in the previous section, in that we shall not be able to obtain indices on the open vertical line segment in Figure 1 connecting $\left(\frac{1}{r}, \frac{1}{q}\right)=(0,0)$ and $\left(0, \frac{1}{2}\right)$ (see Figure 21). Nonetheless, as we shall see, the range that we can obtain is sufficient for proving Theorem 1.1.

As in [7, due to technical difficulties in using the rotational vector fields near $\partial \Omega$ (here $\partial_{\theta}=x_{1} \partial_{2}-x_{2} \partial_{1}$ ), we shall modify the Lebesgue spaces near $\partial \Omega$ from those in (2.15). Specifically, given $0 \leq \gamma<1$, we define

$$
\|h\|_{X_{r, \gamma}}=\|h\|_{L^{s} \gamma(|x|<3 R)}+\|h\|_{L_{|x|}^{r} L_{\theta}^{2}(|x|>2 R)}, \quad \text { with } \gamma=1-\frac{2}{s_{\gamma}} .
$$

We fix $R \geq 1$ large enough so that $\mathcal{K} \subset\{|x|<R\}$. When working with functions on $\mathbb{R}^{2}$, the norms on the right side of (3.2) are taken over $x \in \mathbb{R}^{2}$ with $|x|<3 R$ and $|x|>2 R$ for the first and second terms, respectively. For $\Omega$, we define the norm in the obvious way by extending $h$ to be equal to 0 inside $\mathcal{K}$.

Note that $s_{\gamma}$ in (3.2) is chosen so that $\dot{H}^{\gamma}\left(\mathbb{R}^{2}\right) \subset L^{s_{\gamma}}\left(\mathbb{R}^{2}\right)$ and $\dot{H}^{\gamma}(\Omega) \subset L^{s_{\gamma}}(\Omega)$ by Sobolev embedding. We conclude by Lemma 2.2 of [1] that

$$
\|u\|_{L_{t}^{2} L_{x}^{s \gamma}\left(\mathbb{R}_{+} \times \mathbb{R}^{2}:|x|<3 R\right)} \lesssim\|f\|_{\dot{H}^{\gamma}\left(\mathbb{R}^{2}\right)}+\|g\|_{\dot{H}^{\gamma-1}\left(\mathbb{R}^{2}\right)}, \quad 0<\gamma \leq \frac{1}{2} .
$$

Interpolating with energy conservation lets us conclude the same bound with 2 replaced by any $q \in[2, \infty]$. By this and (2.15), we conclude for the Minkowski space case that if $u$ solves (2.14) with forcing term $F \equiv 0$ and if $0<\gamma \leq \frac{1}{2}$, then

$$
\begin{aligned}
\|u\|_{L_{t}^{q} X_{r, \gamma}\left(\mathbb{R}_{+} \times \mathbb{R}^{2}\right)}+\|u\|_{L_{t}^{\infty} \dot{H}^{\gamma}\left(\mathbb{R}_{+} \times \mathbb{R}^{2}\right)}+\left\|\partial_{t} u\right\|_{L_{t}^{\infty} \dot{H}^{\gamma-1}\left(\mathbb{R}_{+} \times \mathbb{R}^{2}\right)} & \\
& \lesssim\|f\|_{\dot{H}^{\gamma}\left(\mathbb{R}^{2}\right)}+\|g\|_{\dot{H}^{\gamma-1}\left(\mathbb{R}^{2}\right)}
\end{aligned}
$$

assuming that $q, r$ and $\gamma$ are as in (2.15).

Using this estimate, the finite propagation speed for $\square$, and the aforementioned Sobolev inequalities, we see that we also have a local in time variant of this estimate for $\Omega$. Precisely, if $u$ solves the Dirichlet-wave equation (3.1) with forcing term $F \equiv 0$, then

$$
\begin{aligned}
\|u\|_{L_{t}^{q} X_{r, \gamma}([0,1] \times \Omega)}+\|u\|_{L_{t}^{\infty} \dot{H}^{\gamma}([0,1] \times \Omega)}+\left\|\partial_{t} u\right\|_{L_{t}^{\infty} \dot{H}^{\gamma-1}([0,1] \times \Omega)} & \\
& \lesssim\|f\|_{\dot{H}^{\gamma}(\Omega)}+\|g\|_{\dot{H}^{\gamma-1}(\Omega)}
\end{aligned}
$$

with the same assumptions on $q, r$ and $\gamma$.

We shall be able to use (3.3) and (3.4) to prove global variants of some of the estimates in (3.4) due to the fact that we have local energy decay estimates for the 
Dirichlet-wave equation (3.1). Specifically, given fixed $R_{0}>0$ we have

$$
\begin{aligned}
\int_{0}^{\infty}\|u(t, \cdot)\|_{H^{1}\left(|x|<R_{0}\right)}^{2}+\left\|\partial_{t} u(t, \cdot)\right\|_{L^{2}\left(|x|<R_{0}\right)}^{2} d t & \\
& \lesssim\|f\|_{H^{1}}^{2}+\|g\|_{L^{2}}^{2}+\int_{0}^{\infty}\|F(s, \cdot)\|_{L^{2}}^{2} d s,
\end{aligned}
$$

assuming that $\mathcal{K}$ is nonempty and nontrapping, and that $f(x), g(x)$ and $F(t, x)$ all vanish when $|x|>R_{0}$. This was called "Hypothesis 1.1" in [7]. As noted there, it follows from results of Vainberg [15, but another proof can be found in Burq [1. Also, Ralston showed in 10, that this estimate need not hold for Neumann boundary conditions in 2-dimensions, which explains why we are only treating the Dirichlet case in this paper.

Since we have (3.3)-(3.5), we can invoke Theorem 1.4 from 7 to conclude that we have global versions of (3.4) in the special case where $\gamma=\frac{1}{2}$. Precisely, if $u$ solves (3.1) with $F \equiv 0$, then

$$
\begin{aligned}
\|u\|_{L_{t}^{q} X_{r, \frac{1}{2}}\left(\mathbb{R}_{+} \times \Omega\right)}+\|u\|_{L_{t}^{\infty} \dot{H}^{\frac{1}{2}}\left(\mathbb{R}_{+} \times \Omega\right)}+\left\|\partial_{t} u\right\|_{L_{t}^{\infty} \dot{H}^{-\frac{1}{2}}\left(\mathbb{R}_{+} \times \Omega\right)} & \\
& \lesssim\|f\|_{\dot{H}^{\frac{1}{2}}(\Omega)}+\|g\|_{\dot{H}^{-\frac{1}{2}}(\Omega)},
\end{aligned}
$$

assuming the following conditions on $q$ and $r$ :

$$
q>2, \quad \frac{1}{2}=1-\frac{2}{r}-\frac{1}{q}, \quad \text { and } \quad \frac{1}{q}+\frac{1}{r}<\frac{1}{2} .
$$

A limitation of Theorem 1.4 in [7] (which seems difficult to overcome) is that for $n=2$ it applies only to the case of $\gamma=\frac{1}{2}$, whereas for our existence proof we seek estimates with $0<\gamma<\frac{1}{2}$. We get around this problem by an interpolation argument. Note that, by Sobolev embedding and energy conservation, if $0<\gamma<1$ and $s_{\gamma}$ is as in (3.2), then

$$
\begin{aligned}
&\|u\|_{L_{t}^{\infty} L_{x}^{s \gamma}\left(\mathbb{R}_{+} \times \Omega\right)}+\|u\|_{L_{t}^{\infty} \dot{H}^{\gamma}\left(\mathbb{R}_{+} \times \Omega\right)}+\left\|\partial_{t} u\right\|_{L_{t}^{\infty} \dot{H}^{\gamma-1}\left(\mathbb{R}_{+} \times \Omega\right)} \\
& \lesssim\|f\|_{\dot{H}^{\gamma}(\Omega)}+\|g\|_{\dot{H}^{\gamma-1}(\Omega)} \\
&
\end{aligned}
$$

Since $s_{\gamma} \geq 2$, it follows by Hölder's inequality for $S^{1}$ that the $L^{s_{\gamma}}(\Omega)$ norm majorizes the $X_{s_{\gamma}, \gamma}(\Omega)$ norm. Consequently, by the preceding inequality we have that

$$
\begin{aligned}
\|u\|_{L_{t}^{\infty} X_{s_{\gamma}, \gamma}\left(\mathbb{R}_{+} \times \Omega\right)}+\|u\|_{L_{t}^{\infty} \dot{H}^{\gamma}\left(\mathbb{R}_{+} \times \Omega\right)}+\left\|\partial_{t} u\right\|_{L_{t}^{\infty} \dot{H}^{\gamma-1}\left(\mathbb{R}_{+} \times \Omega\right)} & \\
\lesssim\|f\|_{\dot{H}^{\gamma}(\Omega)}+\|g\|_{\dot{H}^{\gamma-1}(\Omega)}, \quad 0<\gamma<1, &
\end{aligned}
$$

if $u$ solves (3.1) with forcing term $F \equiv 0$.

In Figure 2, this corresponds to the exponents on the (open) horizontal line segment corresponding to $\frac{1}{q}=0$. The global estimates (3.6) correspond to the (half-open) segment where $\frac{1}{q}=\frac{1}{2}-\frac{2}{r}$ and $\gamma=\frac{1}{2}$ in this figure. Since the convex hull of this line segment and the horizontal segment is the shaded region in Figure 2. we conclude by interpolating between (3.6) and (3.7) that, for $u$ solving (3.1) with vanishing forcing term, we have

$$
\|u\|_{L_{t}^{q} X_{r, \gamma}\left(\mathbb{R}_{+} \times \Omega\right)}+\|u\|_{L_{t}^{\infty} \dot{H}^{\gamma}\left(\mathbb{R}_{+} \times \Omega\right)}+\left\|\partial_{t} u\right\|_{L_{t}^{\infty} \dot{H}^{\gamma-1}(\Omega)} \lesssim\|f\|_{\dot{H}^{\gamma}(\Omega)}+\|g\|_{\dot{H}^{\gamma-1}(\Omega)},
$$




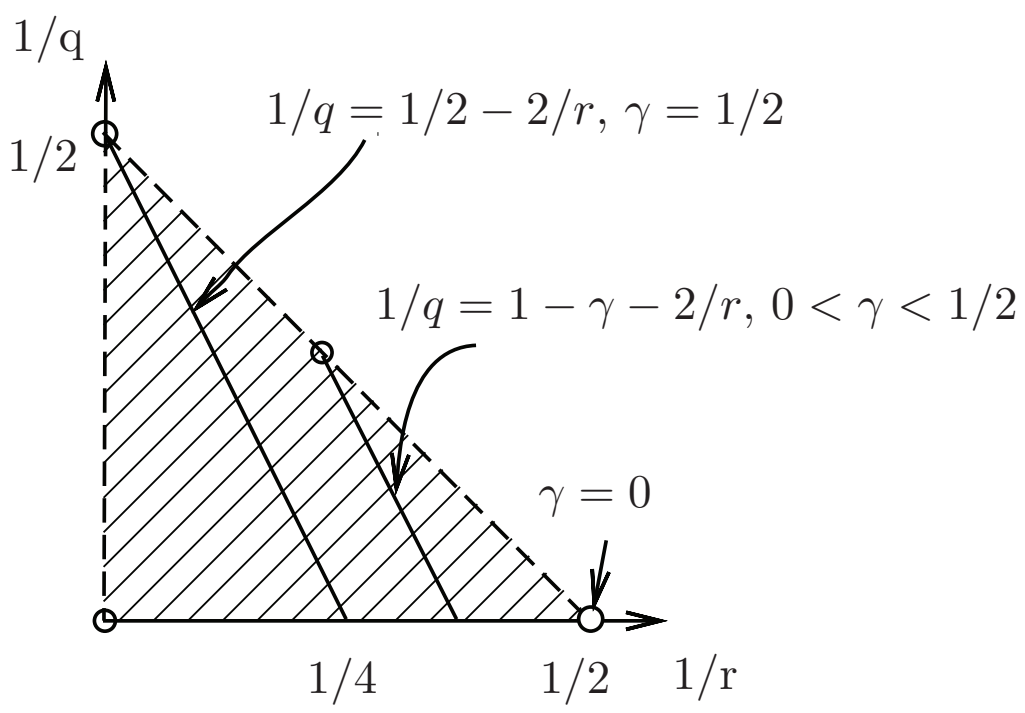

Figure 2. Obstacle case exponents

provided that

$$
q, r>2, \quad r<\infty, \quad \gamma=1-\frac{2}{r}-\frac{1}{q}, \quad \text { and } \quad \frac{1}{q}+\frac{1}{r}<\frac{1}{2} .
$$

By the Christ-Kiselev lemma, if in addition $\tilde{q}$ and $\tilde{r}$ satisfy the variant of (3.9) corresponding to $1-\gamma$,

$$
\tilde{q}, \tilde{r}>2, \quad \tilde{r}<\infty, \quad 1-\gamma=1-\frac{2}{\tilde{r}}-\frac{1}{\tilde{q}}, \quad \text { and } \quad \frac{1}{\tilde{q}}+\frac{1}{\tilde{r}}<\frac{1}{2},
$$

then if $u$ solves the linear Dirichlet-wave equation (3.1) with forcing term $F$, we have

$$
\begin{aligned}
\|u\|_{L_{t}^{\infty} \dot{H}^{\gamma}\left(\mathbb{R}_{+} \times \Omega\right)}+\left\|\partial_{t} u\right\|_{L_{t}^{\infty} \dot{H}^{\gamma-1}\left(\mathbb{R}_{+} \times \Omega\right)}+\|u\|_{L_{t}^{q} X_{r, \gamma}\left(\mathbb{R}_{+} \times \Omega\right)} & \\
& \lesssim\|f\|_{\dot{H}^{\gamma}(\Omega)}+\|g\|_{\dot{H}^{\gamma-1}(\Omega)}+\|F\|_{L_{t}^{\tilde{q}^{\prime} X_{\tilde{r}, 1-\gamma}^{\prime}}}\left(\mathbb{R}_{+} \times \Omega\right)
\end{aligned} .
$$

Here, $X_{\tilde{r}, 1-\gamma}^{\prime}$ denotes the norm which is dual to that of $X_{\tilde{r}, 1-\gamma}$. For the purposes of our existence proof we do not need the exact expression for this dual norm, but we use only the following inequality. If $h=h_{1}+h_{2}$, and $h_{1}=0$ for $|x|>3 R$, respectively $h_{2}=0$ for $|x|<2 R$, then

$$
\|h\|_{X_{\tilde{r}, 1-\gamma}^{\prime}} \leq\left\|h_{1}\right\|_{L^{s_{1-\gamma}^{\prime}(|x|<3 R)}}+\left\|h_{2}\right\|_{L_{|x|}^{\tilde{r}^{\prime}} L_{\theta}^{2}(|x|>2 R)},
$$

where $s_{1-\gamma}^{\prime}$ and $\tilde{r}^{\prime}$ denote the exponents which are conjugate to $s_{1-\gamma}$ and $\tilde{r}$, respectively. In particular, if $\phi$ and $\psi$ are smooth functions, with $\phi+\psi=1$, and

$$
\phi(x)= \begin{cases}1, & |x|<2 R, \\ 0, & |x|>3 R,\end{cases}
$$


then

$$
\|h\|_{X_{\tilde{r}, 1-\gamma}^{\prime}} \leq\|\phi h\|_{L^{s_{1-\gamma}^{\prime}(|x|<3 R)}}+\|\psi h\|_{L_{|x|}^{\tilde{r}^{\prime} L_{\theta}^{2}(|x|>2 R)}} .
$$

As with the proof of Glassey's theorem, we need a variant of (3.11) involving the derivatives $\{\Gamma\}=\left\{\partial_{t}, Z\right\}$, where the $\{Z\}$ vector fields are the ones in (1.4). A problem arises in establishing a version of (3.11) with derivatives, however, in that the proof of such estimates on domains with boundary, as in [7], requires local energy decay estimates that hold only for $\gamma=\frac{1}{2}$ in dimension $n=2$. Our approach will be to establish estimates with derivatives for $\gamma=\frac{1}{2}$ and to interpolate with (3.11) to obtain the desired estimates. For the case $\gamma=\frac{1}{2}$, we have the following variant of Lemma 3.3 of [7].

Lemma 3.1. Suppose that $(f, g, F)$ satisfy the Dirichlet compatibility conditions of order $k+\frac{1}{2}$. Then, for even integers $k=0,2,4, \ldots$,

$$
\begin{aligned}
& \sum_{|\alpha| \leq k}\left(\left\|\Gamma^{\alpha} u\right\|_{L_{t}^{\infty} \dot{H}^{\frac{1}{2}}\left(\mathbb{R}_{+} \times \Omega\right)}+\left\|\Gamma^{\alpha} \partial_{t} u\right\|_{L_{t}^{\infty} \dot{H}^{-\frac{1}{2}}\left(\mathbb{R}_{+} \times \Omega\right)}+\left\|\Gamma^{\alpha} u\right\|_{L_{t}^{q} X_{r, \frac{1}{2}}\left(\mathbb{R}_{+} \times \Omega\right)}\right) \\
& \lesssim \sum_{|\alpha| \leq k}\left(\left\|Z^{\alpha} f\right\|_{\dot{H}^{\frac{1}{2}}(\Omega)}+\left\|Z^{\alpha} g\right\|_{\dot{H}^{-\frac{1}{2}}(\Omega)}+\left\|\Gamma^{\alpha} F\right\|_{L_{t}^{\tilde{q}^{\prime} X_{\tilde{r}, \frac{1}{2}}^{\prime}}\left(\mathbb{R}_{+} \times \Omega\right)}\right),
\end{aligned}
$$

where $(q, r)$ and $(\tilde{q}, \tilde{r})$ are as in (3.9) and (3.10) for $\gamma=\frac{1}{2}$.

Remark. The condition on the data is that $\beta f \in H_{D}^{k+\frac{1}{2}}$, where $\beta$ is a compactly supported cutoff to a neighborhood of the boundary, and similarly $\beta g \in$ $H_{D}^{k-\frac{1}{2}}$. The condition on $F$ is that $\beta F \in L_{t}^{\tilde{q}} H_{D}^{k-\frac{1}{2}}$. These imply that for all $t$, $\left(\beta u(t, \cdot), \beta \partial_{t} u(t, \cdot) \in H_{D}^{k+\frac{1}{2}} \times H_{D}^{k-\frac{1}{2}}\right.$, which will be used in elliptic regularity arguments. We will use the fact that, if $f$ satisfies the $H_{D}^{\frac{1}{2}}$ boundary conditions, then $\|f\|_{\dot{H}_{D}^{\frac{1}{2}}(\Omega)} \approx\|f\|_{\dot{H}^{\frac{1}{2}}(\Omega)}$, where the latter is the norm for the space of restrictions of elements in $\dot{H}^{\frac{1}{2}}\left(\mathbb{R}^{2}\right)$.

Proof. The proof of Lemma 3.1 follows closely the proof of Lemma 3.3 of [7, and we focus here on the modifications necessary for the above estimate. Two key estimates needed for the proof are the following. Assuming that $F \equiv 0$ on $|x| \geq 3 R$, then

$$
\|u\|_{L_{t}^{q} X_{r, \frac{1}{2}}}+\|u\|_{L_{t}^{\infty} \dot{H}_{D}^{\frac{1}{2}}}+\left\|\partial_{t} u\right\|_{L_{t}^{\infty} \dot{H}_{D}^{-\frac{1}{2}}} \lesssim\|f\|_{\dot{H}_{D}^{\frac{1}{2}}}+\|g\|_{\dot{H}_{D}^{-\frac{1}{2}}}+\|F\|_{L_{t}^{2} H_{D}^{-\frac{1}{2}}},
$$

and, with no support assumptions on the data, the following holds:

$$
\|u\|_{L_{t}^{\infty} \dot{H}_{D}^{\frac{1}{2}}}+\left\|\partial_{t} u\right\|_{L_{t}^{\infty} \dot{H}_{D}^{-\frac{1}{2}}}+\|\beta u\|_{L_{t}^{2} H_{D}^{\frac{1}{2}}} \lesssim\|f\|_{\dot{H}_{D}^{\frac{1}{2}}}+\|g\|_{\dot{H}_{D}^{-\frac{1}{2}}}+\|F\|_{L_{t}^{\tilde{q}} X_{\tilde{r}, \frac{1}{2}}^{\prime}} .
$$

Estimate (3.14) follows from the estimates (2.8) of [7] and (3.11), and (3.15) follows from (3.14) by duality.

Using (3.14) and (3.15), and the case $k=0$ of (3.13), the argument on pages 2803-2805 of [7] reduces estimate (3.13) to bounding the following quantity by the right hand side of (3.13), where $\beta$ is a compactly supported cutoff to a neighborhood of the obstacle,

$$
\sum_{j \leq k}\left\|\beta \partial_{t}^{j} u\right\|_{L_{t}^{2} H_{D}^{\frac{1}{2}+k-j}}+\sum_{j \leq k+1}\left\|\beta \partial_{t}^{j} u\right\|_{L_{t}^{\infty} H_{D}^{\frac{1}{2}+k-j}} .
$$


We first observe that the Cauchy data for $\partial_{t}^{j} u, j \leq k$, belongs to $H_{D}^{\frac{1}{2}} \times H_{D}^{-\frac{1}{2}}$; this is seen by using the equation to express $\left(\partial_{t}^{j} u(0, \cdot), \partial_{t}^{j+1} u(0, \cdot)\right)$ in terms of powers of $\Delta$ applied to $(f, g, F)$ and by observing that, by Sobolev embedding,

$$
\begin{aligned}
& \sum_{|\alpha| \leq l}\left\|\partial_{t, x}^{\alpha} F\right\|_{L_{t}^{\infty} \dot{H}^{\frac{1}{2}}}+\sum_{|\alpha| \leq l+1}\left\|\partial_{t, x}^{\alpha} F\right\|_{L_{t}^{\infty} \dot{H}^{-\frac{1}{2}}}+\sum_{|\alpha| \leq l}\left\|\partial_{t, x}^{\alpha} F\right\|_{L_{t}^{2} \dot{H}^{\frac{1}{2}}} \\
& \lesssim \sum_{|\alpha| \leq l+1}\left\|\partial_{t, x}^{\alpha} F\right\|_{L_{t}^{\tilde{q}} \dot{H}^{\frac{1}{2}}}+\sum_{|\alpha| \leq l+2}\left\|\partial_{t, x}^{\alpha} F\right\|_{L_{t}^{\tilde{q}} \dot{H}^{-\frac{1}{2}}} \lesssim \sum_{|\alpha| \leq l+2}\left\|\partial_{t, x}^{\alpha} F\right\|_{L_{t}^{\tilde{q}} X_{\frac{1}{2}, r}^{\prime}} .
\end{aligned}
$$

By (3.15), we thus conclude that the following is bounded by the right hand side of (3.13):

$$
\sum_{j \leq k}\left(\left\|\beta \partial_{t}^{j} u\right\|_{L_{t}^{2} H_{D}^{\frac{1}{2}}}+\left\|\beta \partial_{t}^{j} u\right\|_{L_{t}^{\infty} H_{D}^{\frac{1}{2}}}+\left\|\beta \partial_{t}^{j+1} u\right\|_{L_{t}^{\infty} H_{D}^{-\frac{1}{2}}}\right) .
$$

To bound (3.16), it therefore suffices to bound the following quantity by the right hand side of (3.13):

$$
\sum_{l \leq k / 2}\left(\left\|\beta \partial_{t}^{k-2 l} \Delta^{l} u\right\|_{L_{t}^{2} H_{D}^{\frac{1}{2}}}+\left\|\beta \partial_{t}^{k-2 l} \Delta^{l} u\right\|_{L_{t}^{\infty} H_{D}^{\frac{1}{2}}}+\left\|\beta \partial_{t}^{k+1-2 l} \Delta^{l} u\right\|_{L_{t}^{\infty} H_{D}^{-\frac{1}{2}}}\right) .
$$

Here we are using the fact that we need consider only even powers of $\partial_{t}$ in the first term of (3.16) since $k$ is even and since

$$
\left\|\beta \partial_{t}^{j} u\right\|_{L_{t}^{2} H_{D}^{\frac{1}{2}+k-j}}^{2} \leq\left\|\beta \partial_{t}^{j+1} u\right\|_{L_{t}^{2} H_{D}^{\frac{1}{2}+k-(j+1)}}\left\|\beta \partial_{t}^{j-1} u\right\|_{L_{t}^{2} H_{D}^{\frac{1}{2}+k-(j-1)}} .
$$

To bound (3.19) and conclude the proof, we use the equation $\left(\partial_{t}^{2}-\Delta\right) u=F$ to express

$$
\partial_{t}^{k-2 l} \Delta^{l} u=\partial_{t}^{k} u-\sum_{2 j \leq k-2} \partial_{t}^{k-2-2 j} \Delta^{j} F .
$$

The resulting terms on the right may then be bounded in the appropriate norms using (3.18) and (3.17).

The estimate that we shall require for the existence proof is the following. It is valid provided that $\phi, \psi, \tilde{\phi}, \tilde{\phi} \in C^{\infty}\left(\mathbb{R}^{2}\right)$ take values in $[0,1]$, with

$$
\operatorname{supp}(\phi), \operatorname{supp}(\tilde{\phi}) \subset\{|x|<3 R\}, \quad \operatorname{supp}(\psi), \operatorname{supp}(\tilde{\psi}) \subset\{|x|>2 R\}, \quad \tilde{\phi}+\tilde{\psi} \geq 1 .
$$

We will additionally assume that each is a radial function and that $\phi=1$ (respectively $\psi=1$ ) on a neighborhood of the support of $\tilde{\phi}$ (respectively $\tilde{\psi}$ ).

Corollary 3.2. Suppose that $\gamma,(q, r)$ and $(\tilde{q}, \tilde{r})$ are as in (3.9) and (3.10). Suppose also that $F$ satisfies the Dirichlet compatibility conditions of order $1+\gamma$. Then for the solutions $u$ of (3.1) with vanishing Cauchy data,

$$
\begin{aligned}
\sum_{|\alpha| \leq 1}\left(\left\|Z^{\alpha} u\right\|_{L_{t}^{\infty} L_{x}^{s \gamma}}+\| \psi\right. & \left.\Gamma^{\alpha} u\left\|_{L_{t}^{q} L_{|x|}^{r} L_{\theta}^{2}}+\right\| \phi \Gamma^{\alpha} u \|_{L_{t}^{q} L_{x}^{s} \gamma}\right) \\
& \lesssim \sum_{|\alpha| \leq 1}\left(\left\|\tilde{\psi} \Gamma^{\alpha} F\right\|_{L_{t}^{\tilde{q}} L_{|x|}^{\tilde{r}} L_{\theta}^{2}}+\left\|\tilde{\phi} \Gamma^{\alpha} F\right\|_{L_{t}^{\tilde{q}} L_{x}^{s_{1}^{\prime}}}\right),
\end{aligned}
$$

where all norms are taken over $\mathbb{R}_{+} \times \Omega$. 
Proof. Estimate (3.21) is obtained by interpolating estimate (3.13), which requires $\gamma=\frac{1}{2}$ but allows arbitrarily high order powers of $\Gamma$, with estimate (3.11), which holds for all $0<\gamma<1$, but with 0 powers of $\Gamma$. We thus need to justify the interpolation step by expressing the norms in terms of analytic scales of spaces. We start by noting that

$$
\sum_{|\alpha| \leq 1}\left\|\phi \Gamma^{\alpha} u\right\|_{L_{t}^{q} L_{x}^{s \gamma}} \approx \sum_{|\alpha| \leq 1}\left\|\phi \partial_{t, x}^{\alpha} u\right\|_{L_{t}^{q} L_{x}^{s \gamma}}
$$

and

$$
\sum_{|\alpha| \leq 1}\left\|\psi \Gamma^{\alpha} u\right\|_{L_{t}^{q} L_{|x|}^{r} L_{\theta}^{2}} \approx \sum_{|\alpha| \leq 1}\left\|\psi \partial_{t, r, \theta}^{\alpha} u\right\|_{L_{t}^{q} L_{|x|}^{r} L_{\theta}^{2}}
$$

with similar equalities for the norms in $F$. By embedding $\Omega \cap\{|x|<3 R\}$ in a compact manifold with boundary, the first norm is dominated by (with a different choice of $\phi$ )

$$
\sum_{|\alpha| \leq 1}\left\|D^{\alpha}(\phi u)\right\|_{L_{t}^{q} L_{x}^{s \gamma}\left(\mathbb{R} \times \Omega^{\prime}\right)}
$$

which is a Sobolev norm on a mixed-norm space. That the fractional order Sobolev norms

$$
\left\|\left(1-\partial_{t}^{2}-\Delta_{x}\right)^{\sigma / 2} u\right\|_{L_{t}^{q} L_{x}^{s \gamma}\left(\mathbb{R} \times \Omega^{\prime}\right)}
$$

form an analytic scale of spaces, and that norms for integer $\sigma$ coincide with partial derivatives of order up to $\sigma$ belonging to the mixed-norm space, follows from the fact that Calderón-Zygmund operators are bounded in mixed-norm $L^{p}$ spaces, provided that all Lebesgue exponents lie in the range $(1, \infty)$. See Lizorkin $[8$ for the case of $\mathbb{R}^{n}$. The product manifold setting falls under the theory of UMD spaces; see, for example, [16.

The norms over $\{|x|>2 R\}$ are similarly product norms over polar coordinates. Precisely,

$$
\sum_{|\alpha| \leq 1}\|\psi u\|_{L_{t}^{q} L_{|x|}^{r} L_{\theta}^{2}} \approx\left(\int_{\mathbb{R}}\left(\int_{\mathbb{R}}\left(\int_{S^{1}}|\psi(\rho) u(t, \rho, \theta)|^{2} d \theta\right)^{r / 2}\langle\rho\rangle d \rho\right)^{q / r} d t\right)^{1 / q}
$$

If $W$ denotes the forward solution operator to the wave equation on $\Omega$, in that $u=W F$, then (3.21) can be stated in terms of mapping properties of $\phi W \tilde{\phi}, \phi W \tilde{\psi}$, $\psi W \tilde{\phi}$ and $\psi W \tilde{\psi}$ between such spaces, where the cutoffs $\phi$, etc., may vary from above. For example, we need the bound, for $k=1$,

$$
\sum_{|\alpha| \leq k}\left\|D^{\alpha}(\phi W \tilde{\phi} F)\right\|_{L_{t}^{q} L_{x}^{s \gamma}\left(\mathbb{R} \times \Omega^{\prime}\right)} \lesssim \sum_{|\alpha| \leq k}\left\|D^{\alpha} F\right\|_{L_{t}^{\tilde{q}} L_{x}^{s_{1}^{\prime}-\gamma}\left(\mathbb{R} \times \Omega^{\prime}\right)},
$$

where we may think of $F$ as a function of $(t, x) \in \mathbb{R} \times \Omega^{\prime}$. By (3.12) and Lemma 3.1. this holds for all even integers $k$ provided $\gamma=\frac{1}{2}$, and by (3.11) it holds for $k=0$ for all $0<\gamma<1$. Since the relations (3.9) and (3.10) are linear in the reciprocals of $s_{\gamma}, q$, and $r$ (respectively $s_{1-\gamma}^{\prime}, \tilde{q}$, and $\tilde{r}$ ), we may interpolate to obtain (3.22) for $k=1$ at any point in the shaded region. The estimates for the other terms follow similarly, using the embedding $\dot{H}^{\gamma} \subset L^{s_{\gamma}}$ for the term $\|u\|_{L_{t}^{\infty} L^{s_{\gamma}}}$.

We note that if $.2<\gamma<.5$, as in our application, then it suffices to consider $k \leq 4$ for the estimate (3.13), since one may take the other endpoint with $k=0$ arbitrarily close to the lower right corner. 
Corollary 3.2 gives us the required estimates for the inhomogeneous equation, but we also need the following estimates for the homogeneous wave equation.

Corollary 3.3. Suppose that $\gamma$ and $(q, r)$ are as in (3.9). Suppose also that $\left.f\right|_{\partial \Omega}=$ 0 . Then for the solutions $u$ of (3.1) with $F \equiv 0$,

$$
\begin{aligned}
\sum_{|\alpha| \leq 1}\left(\left\|Z^{\alpha} u\right\|_{L_{t}^{\infty} L_{x}^{s \gamma}}+\| \psi\right. & \left.\Gamma^{\alpha} u\left\|_{L_{t}^{q} L_{|x|}^{r} L_{\theta}^{2}}+\right\| \phi \Gamma^{\alpha} u \|_{L_{t}^{q} L_{x}^{s \gamma}}\right) \\
& \lesssim \sum_{|\alpha| \leq 2}\left\|Z^{\alpha} f\right\|_{L_{1-\gamma(\Omega)}^{s_{1}^{\prime}}}+\sum_{|\alpha| \leq 1}\left\|Z^{\alpha} g\right\|_{L_{1-\gamma}^{s_{1}^{\prime}(\Omega)}} .
\end{aligned}
$$

This is the one step in the proof of Theorem 1.1 where condition (1.5) is used. Indeed, one can replace (1.5) by any norm condition which implies that the left hand side of (3.23) is sufficiently small (where $\gamma=\gamma_{p}$ ). The norms we are using for the initial data are stronger than the norms in (2.19) using inhomogeneous Sobolev spaces, since

$$
W^{1, s_{1-\gamma}^{\prime}} \subset \dot{H}^{\gamma}, \quad L^{s_{1-\gamma}^{\prime}} \subset \dot{H}^{\gamma-1} .
$$

Note also that $s_{1-\gamma_{p}}^{\prime}=q_{p}$, where $q_{p}$ is as in (1.5). We use the above norm due to the difficulty in showing that $\sum_{|\alpha| \leq k}\left\|Z^{\alpha} f\right\|_{\dot{H}^{\gamma}(\Omega)}$ defines an interpolation scale of spaces, simultaneously in $k$ and $\gamma$.

Because of (3.24) (see (1.6) ), we immediately find that when $F \equiv 0,(3.8)$ and (3.13) respectively imply the somewhat weaker versions,

$$
\|u\|_{L_{t}^{\infty} L^{s \gamma}}+\|u\|_{L_{t}^{q} X_{r, \gamma}} \lesssim \sum_{|\alpha| \leq 1}\left\|Z^{\alpha} f\right\|_{L_{1-\gamma}^{s_{1}^{\prime}}}+\|g\|_{L^{s_{1-\gamma}^{\prime}}},
$$

and, for $k=0,2,4, \ldots$ and $(f, g)$ satisfying the compatibility conditions of order $k+\frac{1}{2}$,

$$
\sum_{|\alpha| \leq k}\left(\left\|\Gamma^{\alpha} u\right\|_{L_{t}^{\infty} L^{4}}+\left\|\Gamma^{\alpha} u\right\|_{L_{t}^{q} X_{r, \frac{1}{2}}}\right) \lesssim \sum_{|\alpha| \leq k+1}\left\|Z^{\alpha} f\right\|_{L^{\frac{4}{3}}}+\sum_{|\alpha| \leq k}\left\|Z^{\alpha} g\right\|_{L^{\frac{4}{3}}}
$$

These are the inequalities that we use in the interpolation argument to get (3.23).

The interpolation arguments are similar to those used to prove the inhomogeneous estimate (3.21). For example, for $f$ we have

$$
\sum_{|\alpha| \leq 2}\left\|Z^{\alpha} f\right\|_{L^{s_{1-\gamma}^{\prime}(\Omega)}} \approx \sum_{|\alpha| \leq 2}\left\|\tilde{\psi} Z^{\alpha} f\right\|_{L_{1-\gamma(\Omega)}^{s_{1}^{\prime}}}+\sum_{|\alpha| \leq 2}\left\|\tilde{\phi} Z^{\alpha} f\right\|_{L_{1-\gamma}^{s_{1}^{\prime}(\Omega)}}
$$

and

$$
\begin{aligned}
& \sum_{|\alpha| \leq 2}\left\|\tilde{\psi} Z^{\alpha} f\right\|_{L_{1-\gamma}^{s_{1}^{\prime}(\Omega)}} \approx \sum_{|\alpha| \leq 2}\left\|\tilde{\psi} \partial_{r, \theta}^{\alpha} f\right\|_{L_{1-\gamma}^{s_{1}^{\prime}(\Omega)}}, \\
& \sum_{|\alpha| \leq 2}\left\|\tilde{\phi} Z^{\alpha} f\right\|_{L_{1-\gamma(\Omega)}^{s_{1}^{\prime}}} \approx \sum_{|\alpha| \leq 2}\left\|\tilde{\phi} \partial_{x}^{\alpha} f\right\|_{L_{1-\gamma(\Omega)}^{s_{1}^{\prime}}} .
\end{aligned}
$$

The term $\sum_{|\alpha| \leq 2}\left\|\tilde{\phi} \partial_{x}^{\alpha} f\right\|_{L^{s_{1-\gamma}^{\prime}(\Omega)}}$ can be bounded from above and below by

$$
\|\tilde{\phi} f\|_{W^{2, s_{1-\gamma}^{\prime}}}=\sum_{|\alpha| \leq 2}\left\|\partial_{x}^{\alpha}(\tilde{\phi} f)\right\|_{L_{1-\gamma}^{s_{1}^{\prime}}}
$$

(with different choices of $\tilde{\phi}$ ), which is a standard Sobolev space norm. 
If $U$ denotes the solution operator to the wave equation (3.1) on $\Omega$, with $F, g \equiv 0$, then (3.23) for this special case can be restated in terms of mapping properties of $\phi U \tilde{\phi}, \phi U \tilde{\psi}, \psi U \tilde{\phi}$ and $\psi U \tilde{\psi}$ between these spaces. For example, we need the bound, for $k=1$,

$$
\sum_{|\alpha| \leq k}\left\|\partial_{t, x}^{\alpha}(\phi U \tilde{\phi} f)\right\|_{L_{t}^{q} L_{x}^{s \gamma}\left(\mathbb{R} \times \Omega^{\prime}\right)} \lesssim \sum_{|\alpha| \leq k+1}\left\|\partial_{x}^{\alpha} f\right\|_{L_{x}^{s_{1-\gamma}^{\prime}}\left(\Omega^{\prime}\right)},
$$

where we may think of $f$ as a function of $x \in \Omega^{\prime}$. By (3.26) this holds for all even integers $k$ provided $\gamma=\frac{1}{2}$, and by (3.25) it holds for $k=0$ for all $0<\gamma<1$. Since the relation (3.9) is linear in the reciprocals of $s_{\gamma}, q$, and $r$, we may interpolate to obtain (3.27) for $k=1$ at any point in the shaded region of Figure 2.

Therefore, we conclude the estimate (3.23) is valid in the special case where $g \equiv 0$. Since similar arguments apply to the case where $f \equiv 0$, we get (3.23)

We shall now show how we can use (3.21) and (3.23) to prove our existence results.

Proof of Theorem 1.1. As in our proof of Glassey's theorem, it suffices to consider the case of $p_{c}<p<5$. The proof in the obstacle case requires more care in selecting the indices $q$ and $r$, since the case $r=1$ is not allowed in (3.21), as opposed to its free-space variant (2.15). We thus need to check that we can choose exponents whose ratio is $p$ so that we have estimates that iterate well for equations such as $\square u=|u|^{p}$.

To do this, assume a given $p$ such that $p_{c}<p<5$. We will take $\gamma=\gamma_{p}$ to be the scaling index for $\square u=|u|^{p}$,

$$
\gamma_{p}=1-\frac{2}{p-1}
$$

so that $\frac{5-\sqrt{17}}{4}<\gamma_{p}<\frac{1}{2}$. As noted before, the condition $\frac{1}{2}-\gamma_{p}<\frac{1}{p}$ is equivalent to the condition $p>p_{c}$. This tells us that

$$
\frac{1}{2}-\gamma_{p}<\frac{1}{r} \quad \text { if } \quad p<r<p+\delta(p),
$$

for small $\delta(p)>0$. We fix such an $r$, and determine $\tilde{r}$ by setting $\tilde{r}^{\prime}=r / p$. Since we may assume $\delta(p)<p$, then $p<r<2 p$ so that $\tilde{r} \in(2, \infty)$.

The equality in conditions (3.9) and (3.10) determines that, with $\gamma=\gamma_{p}$,

$$
q\left(\gamma_{p}, r\right)=\frac{p-1}{2} \cdot \frac{r}{r-(p-1)}, \quad\left[\tilde{q}\left(1-\gamma_{p}, \tilde{r}\right)\right]^{\prime}=\frac{p-1}{2} \cdot \frac{\tilde{r}^{\prime}}{p \tilde{r}^{\prime}-(p-1)} .
$$

Since $\tilde{r}^{\prime}=r / p$, we have

$$
\left[\tilde{q}\left(1-\gamma_{p}, \tilde{r}\right)\right]^{\prime}=q\left(\gamma_{p}, r\right) / p
$$

The last inequalities in (3.9) and (3.10) are then equivalent to the conditions

$$
\frac{1}{2}-\gamma_{p}<\frac{1}{r} \text { and } \gamma_{p}-\frac{1}{2}<\frac{1}{\tilde{r}} \text {. }
$$

The first condition is satisfied by (3.28), and the second is satisfied since $\gamma_{p}<\frac{1}{2}$.

To conclude the verification of (3.9)-(3.10), we check that $2<q, r, \tilde{q}, \tilde{r}<\infty$. By construction this holds for $r, \tilde{r}$. We next observe that $q\left(\gamma_{p}, r\right)$ is a decreasing function of $r$ for $r>p$ and that

$$
p+1<q\left(\gamma_{p}, p\right)<2 p .
$$


The first inequality here is equivalent to $p^{2}-3 p-2>0$ and the second to $p<5$. Taking $\delta(p)$ smaller if necessary, it follows that $q\left(\gamma_{p}, r\right) \in(p, 2 p) \subset(2, \infty)$, and hence $q\left(1-\gamma_{p}, \tilde{r}\right) \in(2, \infty)$ by (3.29).

With this choice of indices, we then have the following case of (3.21), valid for solutions $u$ with vanishing Cauchy data:

$$
\begin{aligned}
\sum_{|\alpha| \leq 1}\left(\left\|\psi \Gamma^{\alpha} u\right\|_{L_{t}^{q} L_{|x|}^{r} L_{\theta}^{2}}+\left\|\phi \Gamma^{\alpha} u\right\|_{L_{t}^{q} L_{x}^{s \gamma_{p}}}+\left\|Z^{\alpha} u_{k}\right\|_{L_{t}^{\infty} L_{x}^{s \gamma_{p}}}\right) \\
\quad \lesssim \sum_{|\alpha| \leq 1}\left(\left\|\tilde{\psi} \Gamma^{\alpha} \square u\right\|_{L_{t}^{q / p} L_{|x|}^{r / p} L_{\theta}^{2}}+\left\|\tilde{\phi} \Gamma^{\alpha} \square u\right\|_{L_{t}^{q / p} L_{x}^{s_{1}^{\prime}-\gamma_{p}}}\right) .
\end{aligned}
$$

We now assume that the Cauchy data $(f, g)$ satisfies the smallness condition (1.5) (where $q_{p}$ is the same as our notation $s_{1-\gamma_{p}}^{\prime}$ ), and let $u_{0}$ solve the Cauchy problem (3.1) with forcing term $F \equiv 0$. We then iteratively define $u_{k}, k=1,2,3, \ldots$, by requiring that it solves the equation

$$
\left\{\begin{array}{l}
\square u_{k}(t, x)=F_{p}\left(u_{k-1}(t, x)\right), \quad(t, x) \in \mathbb{R}_{+} \times \Omega, \\
u_{k}(t, x)=0, \quad(t, x) \in \mathbb{R}_{+} \times \partial \Omega, \\
\left.u_{k}\right|_{t=0}=f,\left.\quad \partial_{t} u_{k}\right|_{t=0}=g .
\end{array}\right.
$$

Our goal is to show that if $\varepsilon>0$ in (1.5) is small enough, then so is

$$
M_{k}=\sum_{|\alpha| \leq 1}\left(\left\|\psi \Gamma^{\alpha} u_{k}\right\|_{L_{t}^{q} L_{|x|}^{r} L_{\theta}^{2}}+\left\|\phi \Gamma^{\alpha} u_{k}\right\|_{L_{t}^{q} L_{x}^{s \gamma_{p}}}+\left\|Z^{\alpha} u_{k}\right\|_{L_{t}^{\infty} L_{x}^{s \gamma_{p}}}\right)
$$

for every $k=0,1,2, \ldots$, where we fix $r$ and $q=q\left(\gamma_{p}, r\right)$ as in (3.21).

For $k=0$, it follows from (3.23) that $M_{0} \leq C_{0} \varepsilon$, with $C_{0}>1$ a fixed constant. For $k=1,2, \ldots$, we can then use 3.21) and (3.23) to conclude that

$$
\begin{aligned}
M_{k} \leq C_{0} \varepsilon+C_{1} \sum_{|\alpha| \leq 1}\left(\left\|\tilde{\psi} \Gamma^{\alpha} F_{p}\left(u_{k-1}\right)\right\|_{L_{t}^{q / p} L_{|x|}^{r / p} L_{\theta}^{2}(|x|>2 R)}\right. & \left.+\left\|\tilde{\phi} \Gamma^{\alpha} F_{p}\left(u_{k-1}\right)\right\|_{L_{t}^{q / p} L_{x} s_{1-\gamma_{p}}(|x|<3 R)}\right) \\
=C_{0} \varepsilon+C_{1}(I+I I) &
\end{aligned}
$$

with $C_{1}$ another fixed constant. Assuming that $M_{k-1} \leq 2 C_{0} \varepsilon$, we will inductively show that $M_{k} \leq 2 C_{0} \varepsilon$.

We first note that since $s_{\gamma_{p}}=p-1>2$ and $n=2$, it follows from Sobolev embedding on $\Omega$ that

$$
\|v\|_{L^{\infty}} \lesssim \sum_{|\alpha| \leq 1}\left\|\partial^{\alpha} v\right\|_{L^{s \gamma_{p}}}
$$

This means that

$$
\left\|u_{k-1}(t, x)\right\|_{L_{t}^{\infty} L_{x}^{\infty}} \leq C M_{k-1} \leq 2 C C_{0} \varepsilon \leq 1,
$$

provided that $\varepsilon$ is small enough, which verifies the condition on $u$ in (1.2). Our assumption (1.2) on the nonlinear term, $F_{p}$, then implies that

$$
\sum_{|\alpha| \leq 1}\left|\Gamma^{\alpha} F_{p}\left(u_{k-1}\right)\right| \lesssim\left|u_{k-1}\right|^{p-1} \sum_{|\alpha| \leq 1}\left|\Gamma^{\alpha} u_{k-1}\right| .
$$


Since the collection $\{\Gamma\}$ contains $\partial_{\theta}$, by Sobolev embedding on the circle we have

$$
\|v(|x| \cdot)\|_{L_{\theta}^{\infty}} \lesssim \sum_{|\alpha| \leq 1}\left\|\Gamma^{\alpha} v(|x| \cdot)\right\|_{L_{\theta}^{2}}, \quad|x|>2 R .
$$

Consequently, since $\Omega$ contains the set $|x|>2 R$, it follows for fixed $|x|>2 R$ and $t>0$ that

$$
\sum_{|\alpha| \leq 1}\left\|\Gamma^{\alpha} F_{p}\left(u_{k-1}(t,|x| \cdot)\right)\right\|_{L_{\theta}^{2}} \lesssim \sum_{|\alpha| \leq 1}\left\|\Gamma^{\alpha} u_{k-1}(t,|x| \cdot)\right\|_{L_{\theta}^{2}}^{p},
$$

which means that $I \leq C_{2} M_{k-1}^{p}$, for some uniform constant $C_{2}$.

To handle the term $I I$ in (3.31), we note that since $s_{\gamma_{p}}>2$ and $n=2$, it follows from Sobolev embedding on $\Omega \cap\{|x|<3 R\}$ that

$$
\|\tilde{\phi} v\|_{L^{2(p-1)}(|x|<3 R)} \lesssim \sum_{|\alpha| \leq 1}\left\|\phi Z^{\alpha} v\right\|_{L^{s \gamma_{p}}(|x|<3 R)}
$$

Since $s_{1-\gamma}^{\prime}<2$ satisfies

$$
\frac{1}{s_{1-\gamma}^{\prime}}=\frac{1}{2}+\frac{1}{s_{\gamma}}
$$

by Hölder's inequality we have, for each fixed $t$, that

$$
\begin{aligned}
& \sum_{|\alpha| \leq 1}\left\|\tilde{\phi} \Gamma^{\alpha} F_{p}\left(u_{k-1}(t, \cdot)\right)\right\|_{L^{s_{1}^{\prime}-\gamma(|x|<3 R)}} \\
& \lesssim \sum_{|\alpha| \leq 1}\left\|\tilde{\phi} u_{k-1}\right\|_{L^{2(p-1)}(|x|<3 R)}^{p-1} \sum_{|\alpha| \leq 1}\left\|\phi \Gamma^{\alpha} u_{k-1}(t, \cdot)\right\|_{L^{s} \gamma(|x|<3 R)} \\
& \lesssim \sum_{|\alpha| \leq 1}\left\|\phi \Gamma^{\alpha} u_{k-1}(t, \cdot)\right\|_{L^{s \gamma}(|x|<3 R)}^{p} .
\end{aligned}
$$

This implies that we also have $I I \leq C_{3} M_{k-1}^{p}$, for some uniform constant $C_{3}$, which together with the bound for $I$ gives

$$
M_{k} \leq C_{0} \varepsilon+C_{1}\left(C_{2}+C_{3}\right) M_{k-1}^{p} \leq C_{0} \varepsilon+C_{1}\left(C_{2}+C_{3}\right)\left(2 C_{0} \varepsilon\right)^{p} .
$$

Thus, if $\varepsilon$ is sufficiently small, we conclude that

$$
M_{k} \leq 2 C_{0} \varepsilon, \quad k=0,1,2, \ldots
$$

To finish the proof of the existence results, for $p_{c}<p<5$ we need to show that the $u_{k}$ converge to a solution of (1.1). To do this it suffices to show that

$$
A_{k}=\left\|u_{k}-u_{k-1}\right\|_{L_{t}^{\infty} \dot{H}^{\gamma_{p}}}
$$

tends geometrically to zero as $k \rightarrow \infty$. Since

$$
\left|F_{p}(v)-F_{p}(w)\right| \lesssim|v-w|\left(|v|^{p-1}+|w|^{p-1}\right)
$$

when $v$ and $w$ are small, the proof of (3.33) can be adapted to show that, for small $\varepsilon>0$, there is a uniform constant $C$ so that

$$
A_{k} \leq C A_{k-1}\left(M_{k-1}+M_{k-2}\right)^{p-1}
$$

which, by (3.33), implies that $A_{k} \leq \frac{1}{2} A_{k-1}$ for small $\varepsilon$. Since $A_{1}$ is finite, the claim follows, which finishes the proof of the existence results for the range of $p_{c}<p<5$.

As in $\S 2$, the results for $p \geq 5$ in Theorem 1.1 follow from the above and the fact that condition (1.2) becomes weaker as $p$ increases. 


\section{REFERENCES}

[1] N. Burq, Global Strichartz estimates for nontrapping geometries: About an article by H. F. Smith and C. D. Sogge: "Global Strichartz estimates for nontrapping perturbations of the Laplacian", Comm. Partial Differential Equations 25 (2000), 2171-2183. MR1789924 $(2001 \mathrm{j}: 35180)$

[2] M. Christ, A. Kiselev, Maximal functions associated to filtrations, Jour. Func. Anal. 179 (2001), 409-425. MR1809116 (2001i:47054)

[3] Y. Du, J. Metcalfe, C.D. Sogge, and Y. Zhou, Concerning the Strauss conjecture and almost global existence for nonlinear Dirichlet-wave equations in 4-dimensions, Comm. Partial Differential Equations 33 (2008), 1487-1506. MR2450167 (2009k:35195)

[4] D. Fang, C. Wang, Some remarks on Strichartz estimates for homogeneous wave equation, Nonlinear Anal. 65 (2006), no. 3, 697-706. MR2231083 (2007c:35096)

[5] D. Fang, C. Wang, Weighted Strichartz estimates with angular regularity and their applications, Forum Math. 23 (2011), no. 1, 181-205. MR2769870

[6] R. T. Glassey, Existence in the large for $\square u=F(u)$ in two space dimensions, Math. Z. 178 (1981), 233-261. MR631631 (84h:35106)

[7] K. Hidano, J. Metcalfe, H. F. Smith, C. D. Sogge, Y. Zhou, On abstract Strichartz estimates and the Strauss conjecture for nontrapping obstacles, Trans. Amer. Math. Soc. 362 (2010), 2789-2809. MR 2584618

[8] P. I. Lizorkin, Multipliers of Fourier integrals and bounds of convolution in spaces with mixed norms. Applications, Math. USSR Izv., 4 no. 1 (1970), 225-255.

[9] J. Metcalfe, Global Strichartz estimates for solutions to the wave equation exterior to a convex obstacle, Trans. Amer. Math. Soc. 356 (2004), 4839-4855. MR.2084401 (2006d:35141)

[10] J. Ralston, Note on the decay of acoustic waves, Duke Math. J. 46 (1979), 799-804. MR.552527 (80m:35051)

[11] H. F. Smith, C. D. Sogge, Global Strichartz estimates for nontrapping perturbations of the Laplacian, Comm. Partial Differential Equations 25 (2000), 2171-2183. MR.1789924 (2001j:35180)

[12] C. D. Sogge, Lectures on nonlinear wave equations. Second edition. International Press, Boston, MA, 2008. MR2455195 (2009i:35213)

[13] E. M. Stein, G. Weiss, Introduction to Fourier analysis on Euclidean spaces, Princeton Mathematical Series, No. 32. Princeton University Press, Princeton, N.J., 1971. MR 0304972 (46:4102)

[14] J. Sterbenz, Angular regularity and Strichartz estimates for the wave equation, with an appendix by Igor Rodnianski, Int. Math. Res. Not. 2005 No. 4, 187-231. MR2128434 (2006i:35212)

[15] B. R. Vainberg, The short-wave asymptotic behavior of the solutions of stationary problems, and the asymptotic behavior as $t \rightarrow \infty$ of the solutions of nonstationary problems, Russian Math. Surveys 30 (1975), 1-58. MR0415085 (54:3176)

[16] F. Zimmermann, On vector valued Fourier multiplier theorems, Studia Math. 93 (1989), 201-222. MR.1030488 (91b:46031)

Department of Mathematics, University of Washington, Seattle, Washington 98195

Department of Mathematics, Johns Hopkins University, Baltimore, Maryland 21218

Department of Mathematics, Johns Hopkins University, Baltimore, Maryland 21218 